\title{
Recurrent abdominal pain, vomiting, velvet-like changes in the small intestine in a patient with multiple acyl-CoA dehydrogenase deficiency: a case report
}

\author{
Ziqing Ye ${ }^{1}$, Jieru Shi ${ }^{1}$, Xiaolan $\mathrm{Lu}^{1}$, Yingying Meng ${ }^{1}$, Wei Lu ${ }^{2}$, Bingbing $\mathrm{Wu}^{3}$, Ying Huang ${ }^{1}$ \\ ${ }^{1}$ Department of Gastroenterology, Children's Hospital of Fudan University, Shanghai, China; ${ }^{2}$ Department of Pediatric Endocrinology and Inborn \\ Metabolic Diseases, Children's Hospital of Fudan University, Shanghai, China; ${ }^{3}$ Key Laboratory of Birth Defects, Children's Hospital of Fudan \\ University, Shanghai, China \\ Correspondence to: Ying Huang, MD, PhD. Department of Gastroenterology, Children's Hospital of Fudan University, 399 Wanyuan Road, Shanghai \\ 201102, China. Email: yhuang815@163.com.
}

\begin{abstract}
Multiple acyl-CoA dehydrogenase deficiency (MADD) is an inborn error of metabolism in fatty acid oxidation. We described an unusual case of recurrent vomiting and abdominal pain in a child with MADD, presenting with velvet-like changes in the small intestine. Because of prominent gastrointestinal manifestations and small intestine ulcers, the patient was first diagnosed as Crohn's disease. The patient was admitted to our institution because of recurrent symptoms despite treatment. Upper and lower endoscopy, computed tomography and trios exome sequencing were performed. This patient underwent a repeated video endoscopy, which showed velvet-like changes in the small intestine rather than ulcers. Liver steatosis was identified by computed tomography. Serum tandem mass spectrometry showed elevated C8 and C10. Trios exome sequencing revealed compound heterozygous variants of c.250G>A, 524G>T in ETFDH. The diagnosis of MADD was made. Patient responded to oral riboflavin treatment. With this case, we aimed to highlight the importance of tandem mass spectrometry and genetic sequencing, especially when the endoscopic findings are not pathognomonic in pediatric cases with recurrent gastrointestinal complaints. We confirmed the diagnosis with next generation sequencing, and described unusual findings of velvet-like changes mimicking ulcers in the small intestine in this patient with MADD.
\end{abstract}

Keywords: Multiple acyl-CoA dehydrogenase deficiency (MADD); exome sequencing; ETFDH genes; case report

Submitted Aug 20, 2020. Accepted for publication Dec 04, 2020.

doi: $10.21037 / \mathrm{tp}-20-253$

View this article at: http://dx.doi.org/10.21037/tp-20-253

\section{Introduction}

Multiple acyl-CoA dehydrogenase deficiency (MADD) is a ultrarare inborn error of metabolism in fatty acid oxidation and amino acid (1). Patients with later-onset MADD develop progressive myopathy and heterogeneous symptoms of intermittent vomiting and hypoglycemia (2). The endoscopic findings have not been described in detail among patients with MADD. Therefore, we present, for the first time in literature, a MADD patient with recurrent vomiting and abdominal pain and diffuse whitish velvetlike lesions in the small intestine shown in the video capsule endoscopy. The velvet-like lesions might be misdiagnosed as mucosal ulcers considering the distributions and manifestations of the lesions. Here we described one MADD case with compound heterozygous variants of the ETFDH gene. We present the following case in accordance with the CARE checklist (available at http://dx.doi. org/10.21037/tp-20-253).

\section{Case presentation}

All procedures performed in studies involving human participants were in accordance with the ethical standards 


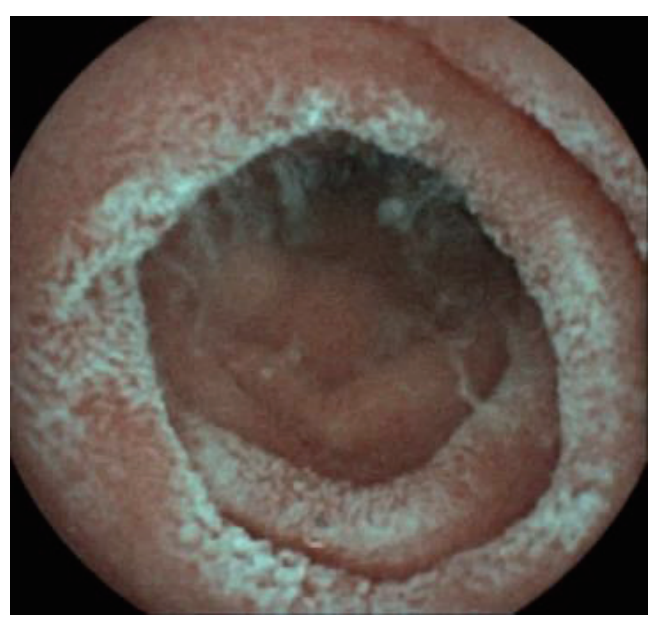

Figure 1 Findings of video capsule endoscopy. Diffuse velvet-like changes are present in multiple segments of the small intestine. There is no apparent ulceration or erosion.

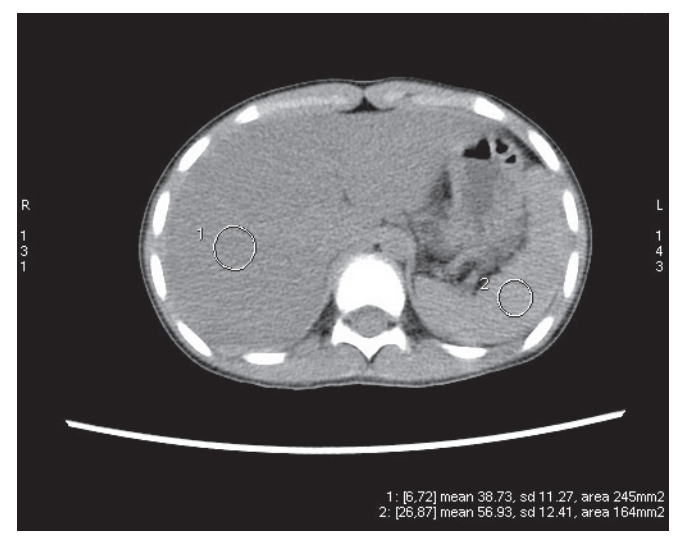

Figure 2 Abdominal computerized tomography scan without contrast. There is hepatic lipomatosis and the liver is not enlarged, and there is no splenomegaly. The computerized tomography value of the liver is lower than that of the spleen.

of the institutional and/or national research committee(s) and with the Helsinki Declaration (as revised in 2013). Written informed consent was obtained from the patient's parents. An 8-year-old girl of non-consanguineous parents from southern China with history of recurrent abdominal pain and vomiting since 4 years of age presented to the gastroenterology department. Colonoscopy was unremarkable, while multiple ulcers were identified in the small intestine on video capsule endoscopy. She was diagnosed with Crohn's disease, and initially treated with exclusive enteral nutrition, mesalamine and azathioprine in a local hospital. She still complained of abdominal pain and had multiple episodes of vomiting and was referred to our unit. Apart from gastrointestinal symptoms, she also reported muscle weakness during attacks.

On presentation, her physical examination was unremarkable. There was no sign of perianal diseases. Inflammatory markers, including C-reactive protein and erythrocyte sedimentation rate were within normal limits. Other laboratory investigations showed a lactate of $2.9 \mathrm{mmol} / \mathrm{L}$, $\mathrm{pH}$ of 7.311 , serum free fatty acid of $2.161 \mathrm{mmol} / \mathrm{L}$. Considering the onset of age and recurrent vomiting, serum tandem mass spectrometry was performed, and showed C10 of $1.29 \mu \mathrm{M}$, C8 of $0.57 \mu \mathrm{M}$, C5/C3 of $0.362 \mu \mathrm{M}$.

Upper endoscopy and colonoscopy were unremarkable. A repeat video capsule endoscopy revealed diffuse whitish velvet-like changes in several segments without apparent mucosal ulcerations (Figure 1). Abdominal computed tomography without contrast showed hepatic lipomatosis (Figure 2). As a result, diagnosis of Crohn's disease cannot be supported in our patient.

Hepatic lipomatosis might not be a usual cause of recurrent abdominal pain and vomiting in children. However, when associated with elevated free fatty acid, lactate and abnormal results of serum tandem mass spectrometry, metabolic disorders are of high suspicion. Endocrinology was consulted. Genomic DNA was extracted from the peripheral whole blood of the patient and parent using the Agilent SureSelectXT Human All Exon 50-Mb kit. Exome sequencing resulted in an average $100 \times$ coverage using the Illumina HiSeq2000/2500 sequencer (Illumina, San Diego, CA, USA). Sequence read alignments were completed using Novoalign (V2.07.18) against the human reference genome GRCh37.p10 (http://www.novocraft.com). The bioinformatics pipeline has been previously described (3). In brief, after quality control, variants were filtered by means of public databases, including Human Gene Mutation Database (HGMD) Professional, the Exome Aggregation Consortium (ExAC), and an in-house database. Sanger sequencing was performed with a Biosystems 3500 DNA Analyzer and analyzed by Mutation Surveyor V4.0.9.

Trios exome sequencing showed that compound heterozygous mutations of c. [250G>A] + [524G>T] were identified in ETFDH, which was confirmed by Sanger sequencing (Figure 3).

This patient was diagnosed with late-onset MADD and treated with riboflavin with a dosage of $100 \mathrm{mg} /$ day instead of mesalamine and azathioprine. She was symptom-free soon after initiation of treatment. In addition, she did not 


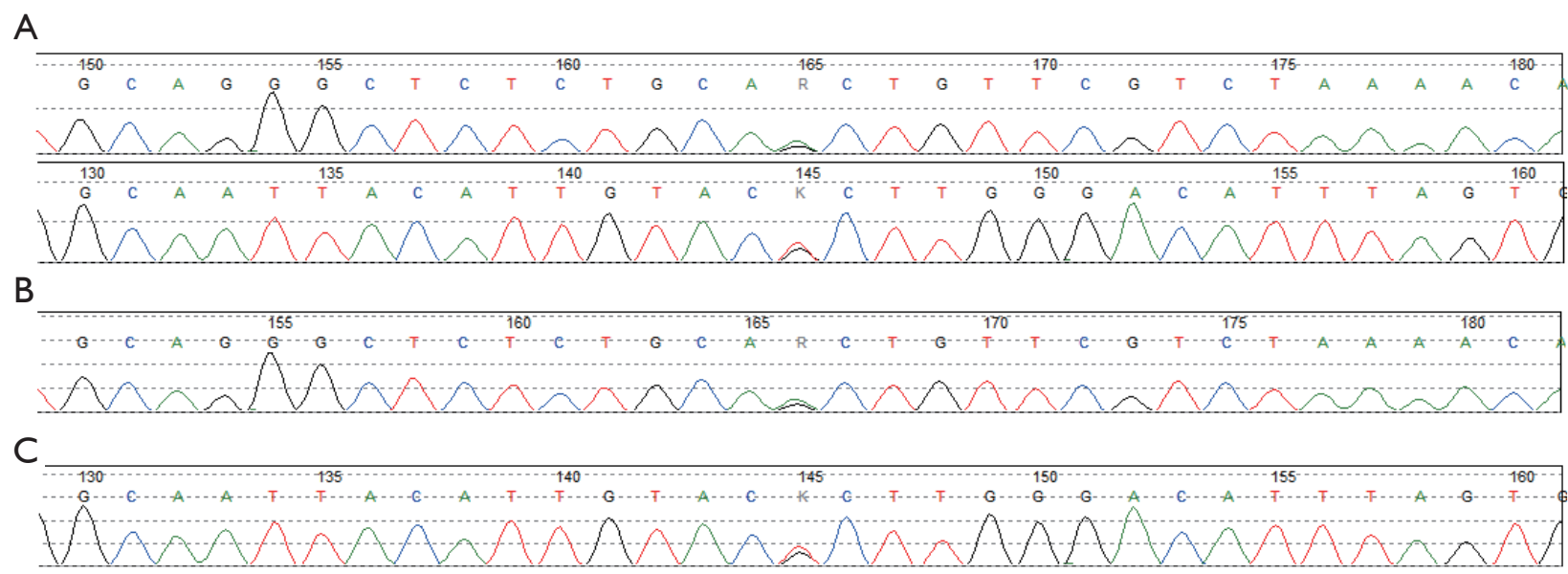

Figure 3 Sanger sequencing of ETFDH confirmed mutations in our patient. (A) Patient: ETFDH c.250G>A, c.524G>T; (B) father of the patient: ETFDH c.250G >A; (C) mother of the patient: ETFDH c.524G>T.

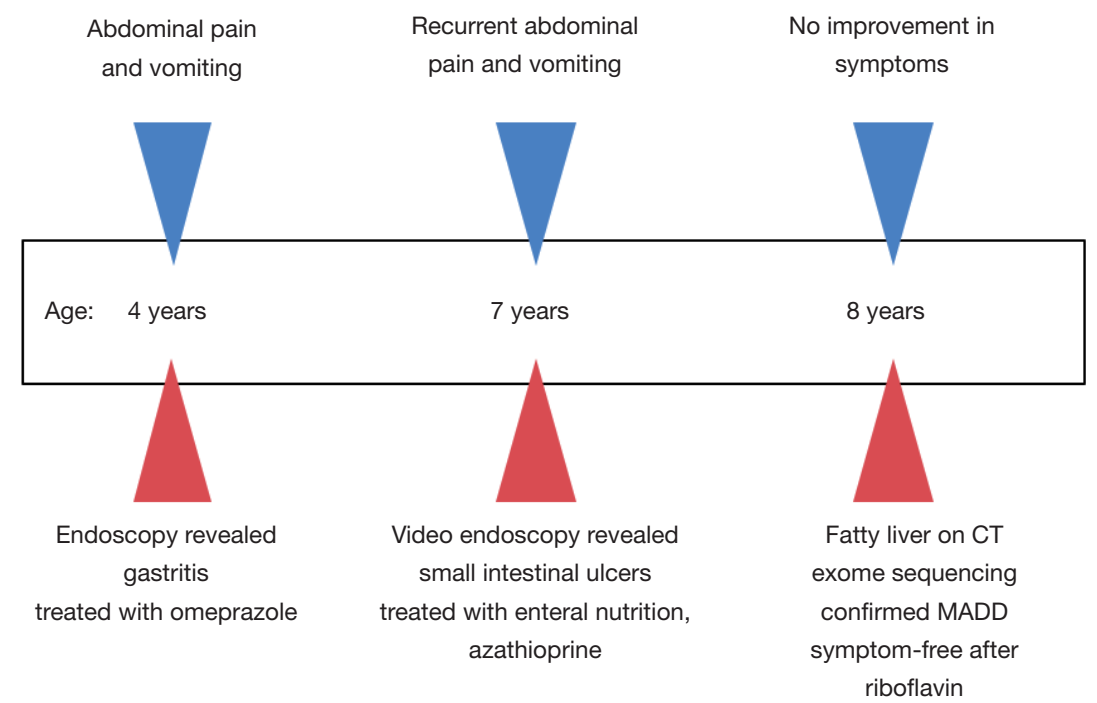

Figure 4 The timeline of this case, including clinical manifestations, tests and treatments. CT, computed tomography; MADD, multiple acyl-CoA dehydrogenase deficiency.

complain about any adverse effects of the treatment. We followed the patient for 2 years, and she did not report any attack of vomiting and abdominal pain. The parent refused endoscopy since the patient did not show any symptoms after treatment. The timeline of this case was shown in Figure 4.

\section{Discussion}

MADD (OMIM 231680) is an autosomal recessive inherited fatty acid and amino-acid metabolism disorder (4). Most late-onset MADD can be corrected by therapeutic dosage of riboflavin, thus is regarded as riboflavin-responsive MADD (5). Patients were diagnosed by Sanger sequencing of ETFDH or whole exome sequencing $(2,6)$. Genetic sequencing is crucial in the diagnosis of MADD, for there is no need to proceed to more invasive diagnostic testing including muscle biopsy (6). The ETFDH gene encodes electron transfer flavoprotein ubiquinone oxidoreductase, which exists as a $64-\mathrm{kDa}$ monomer harboring one molecule of the flavin adenine dinucleotide and a $4 \mathrm{Fe} 4 \mathrm{~S}$ cluster in the inner mitochondrial membrane $(7,8)$. We reported one 
MADD patient presenting with recurrent vomiting and abdominal pain. The patient was diagnosed by trios whole exome sequencing.

Hepatic lipomatosis might be an important extramuscular finding of late-onset MADD, and severe hepatic lipomatosis was revealed by ultrasound in 4 out of 13 patients with MADD (9). Hepatic lipomatosis and lipid storage myopathy in MADD are due to impaired fat oxidation and greater dependence upon carbohydrate oxidation (9). Patients might be asymptomatic. It is generally considered as a benign and stable condition. However, progression to cirrhosis and acute liver failure have been reported among three adult cases of late-onset MADD since 2013 (10-12). Liver involvement is associated with various fatty acid oxidation disorders, and steatosis is a reliable but non-specific sign (13). Therefore, systematic screening is encouraged if there is high index of suspicion.

Endoscopic findings of MADD or other fatty acid oxidation disorder have not been specifically reported yet. Intestinal fat accumulation was described in familial hypobetalipoproteinemia due to apolipoprotein B R463 mutation (14). We speculated that velvet-like lesions in the small intestine were caused by similar pathogenesis of hepatic lipomatosis. However, we could not verify our findings in other MADD patients considering the rarity of the disease and indications for video endoscopy. Because our patient had prominent gastrointestinal complains of vomiting and abdominal pain, while the first video capsule endoscopy showed diffuse whitish changes mimicking ulcerations, she was misdiagnosed as Crohn's disease. However, inborn errors of metabolism should also be included in the differential diagnosis in patients with predominant gastrointestinal complaints, including relatively nonspecific symptoms of recurrent abdominal pain and vomiting (15).

Primary MADD is caused by a genetic defect in electron transfer flavoproteins or in electron-transferring flavoprotein (ETF) dehydrogenase (4). The c.250G>A and c.524G $>\mathrm{T}$ variants in ETFDH have already been reported to be pathogenic $(2,16)$. In addition, the c. $250 \mathrm{G}>\mathrm{A}$ variant might be a possible founder mutation in southern China, and clustered in Fujian Province (2). Our patient also came from this region. The estimated carrier frequency was about $1.35 \%$ among the normal population (2).

Our patient was responsive to riboflavin treatment and remained symptom-free during 2 years of follow-up. The parent refused further endoscopy after treatment as the patient denied having attacks of vomiting or abdominal pain. Therefore, we could not compare MADD patients demonstrate multiorgan dysfunction especially in the case of catabolism (17). D,L-3-hydroxybutyrate treatment is effective in improving clinical symptoms and survival in severely affected MADD cases (1).

\section{Conclusions}

For children presenting with predominant gastrointestinal symptoms of recurrent abdominal pain and vomiting, pediatricians should consider possibility of metabolic disorders, especially when results of imaging and endoscopy are inconclusive. In this setting, tandem mass spectrometry and genetic sequencing should be timely engaged.

\section{Acknowledgments}

We express our gratitude to the patient.

Funding: None.

\section{Footnote}

Reporting Checklist: The authors have completed the CARE reporting checklist. Available at http://dx.doi.org/10.21037/ tp-20-253

Conflicts of Interest: All authors have completed the ICMJE uniform disclosure form (available at https://tp.amegroups. com/article/view/59989/coif). The authors have no conflicts of interest to declare.

Ethical Statement: The authors are accountable for all aspects of the work in ensuring that questions related to the accuracy or integrity of any part of the work are appropriately investigated and resolved. All procedures performed in studies involving human participants were in accordance with the ethical standards of the institutional and/or national research committee(s) and with the Helsinki Declaration (as revised in 2013). Written informed consent was obtained from the patient's parents.

Open Access Statement: This is an Open Access article distributed in accordance with the Creative Commons Attribution-NonCommercial-NoDerivs 4.0 International License (CC BY-NC-ND 4.0), which permits the noncommercial replication and distribution of the article with the strict proviso that no changes or edits are made and the original work is properly cited (including links to both the formal publication through the relevant DOI and the license). 
See: https://creativecommons.org/licenses/by-nc-nd/4.0/.

\section{References}

1. van Rijt WJ, Jager EA, Allersma DP, et al. Efficacy and safety of D,L-3-hydroxybutyrate (D,L-3-HB) treatment in multiple acyl-CoA dehydrogenase deficiency. Genet Med 2020;22:908-16.

2. Wang ZQ, Chen XJ, Murong SX, et al. Molecular analysis of 51 unrelated pedigrees with late-onset multiple acylCoA dehydrogenation deficiency (MADD) in southern China confirmed the most common ETFDH mutation and high carrier frequency of c.250G>A. J Mol Med (Berl) 2011;89:569-76.

3. Yang L, Kong Y, Dong X, et al. Clinical and genetic spectrum of a large cohort of children with epilepsy in China. Genet Med 2019;21:564-71.

4. Olsen RK, Andresen BS, Christensen E, et al. Clear relationship between ETF/ETFDH genotype and phenotype in patients with multiple acyl-CoA dehydrogenation deficiency. Hum Mutat 2003;22:12-23.

5. Xu J, Li D, Lv J, et al. ETFDH Mutations and Flavin Adenine Dinucleotide Homeostasis Disturbance Are Essential for Developing Riboflavin-Responsive Multiple Acyl-Coenzyme A Dehydrogenation Deficiency. Ann Neurol 2018;84:659-73.

6. Fan X, Xie B, Zou J, et al. Novel ETFDH mutations in four cases of riboflavin responsive multiple acylCoA dehydrogenase deficiency. Mol Genet Metab Rep 2018;16:15-9.

7. Schiff M, Froissart R, Olsen RK, et al. Electron transfer flavoprotein deficiency: functional and molecular aspects. Mol Genet Metab 2006;88:153-8.

8. Simkovic M, Degala GD, Eaton SS, et al. Expression of human electron transfer flavoprotein-ubiquinone

Cite this article as: Ye Z, Shi J, Lu X, Meng Y, Lu W, Wu B, Huang Y. Recurrent abdominal pain, vomiting, velvetlike changes in the small intestine in a patient with multiple acyl-CoA dehydrogenase deficiency: a case report. Transl Pediatr 2021;10(1):183-187. doi: 10.21037/tp-20-253 oxidoreductase from a baculovirus vector: kinetic and spectral characterization of the human protein. Biochem J 2002;364:659-67.

9. Zhu M, Zhu X, Qi X, et al. Riboflavin-responsive multiple Acyl-CoA dehydrogenation deficiency in 13 cases, and a literature review in mainland Chinese patients. J Hum Genet 2014;59:256-61.

10. Soldath P, Lund A, Vissing J. Late-onset MADD: a rare cause of cirrhosis and acute liver failure? Acta Myol 2020;39:19-23.

11. Scheicht D, Werthmann ML, Zeglam S, et al. Muscle weakness and early stages of liver failure in a 22-year-old man. Internist (Berl) 2013;54:1016-22.

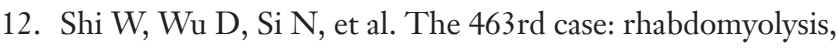
acute kidney failure and acute hepatic failure. Zhonghua Nei Ke Za Zhi 2018;57:381-4.

13. Baruteau J, Sachs P, Broué P, et al. Clinical and biological features at diagnosis in mitochondrial fatty acid betaoxidation defects: a French pediatric study of 187 patients. J Inherit Metab Dis 2013;36:795-803.

14. Noto D, Cefalù AB, Cannizzaro A, et al. Familial hypobetalipoproteinemia due to apolipoprotein B R463W mutation causes intestinal fat accumulation and low postprandial lipemia. Atherosclerosis 2009;206:193-8.

15. Guerrero RB, Kloke KM, Salazar D. Inborn Errors of Metabolism and the Gastrointestinal Tract. Gastroenterol Clin North Am 2019;48:183-98.

16. Liang WC, Ohkuma A, Hayashi YK, et al. ETFDH mutations, CoQ10 levels, and respiratory chain activities in patients with riboflavin-responsive multiple acylCoA dehydrogenase deficiency. Neuromuscul Disord 2009;19:212-6.

17. Olpin SE. Implications of impaired ketogenesis in fatty acid oxidation disorders. Prostaglandins Leukot Essent Fatty Acids 2004;70:293-308. 